\title{
The Compressed Gas Thermodynamics Processes on Gas Pipelines
}

\author{
Saif A. Mouhammad \\ Physics Department, Faculty of Science, Taif University, Taif, Kingdom of Saudi Arabia \\ Email: saifnet70@hotmail.com
}

Received 20 February 2016; accepted 21 March 2016; published 24 March 2016

Copyright (C) 2016 by author and Scientific Research Publishing Inc.

This work is licensed under the Creative Commons Attribution International License (CC BY). http://creativecommons.org/licenses/by/4.0/

c) (i) Open Access

\begin{abstract}
Transport of gas through pipelines is the most energy-intensive production. About $80 \%$ of the energy of the whole oil and gas production is used for the gas transportation through pipelines. To that end, enhancement of the efficiency and energy saving in the field of gas transport are solved with the aid of the basic thermodynamic relations which are mainly presented in this article.
\end{abstract}

\section{Keywords}

Transportation of Gas, Compression, Potential Gas Work, Gas Flow Work, Pseudo-Provided Parameters

\section{Introduction}

Taking into account that the transport of gas through pipelines is a costly process in the oil and gas production, the article pays great attention to the main stage of the process, the compression of gas by the gas pumping units mounted at compressor stations (CS) located along the pipeline every tens of kilometres depending on the performance of the pipeline. In this regard, the article presents the calculated thermodynamic ratios for gas compression by gas compressor units based on the laws of thermodynamics for reversible and irreversible processes - thermodynamics by Professor N. I. Belokon [1], in contrast to the classical thermodynamics. Gas centrifugal compressors are primarily used as the gas pumping units for industrial conditions and of the large capacity (for example, 10 million $\mathrm{m}^{3}$ per day and more) [2].

\section{Initial Concepts}

The thermodynamic natural gas compression processes represent the main process of gas transport through pipelines. Gas compression is a costly process as it accounts for about $80 \%$ of the energy consumption of all the oil 
and gas production (drilling, development, exploitation wells in the oil fields, integrated oil and gas units for transport; transport, processing and sale of the product). In this context, in order to save energy when gas is compressed and choose an energy-efficient technology for gas transport, it is necessary to calculate the rational and efficient characteristics of the transport process to ensure reliable and safe operation of the pipeline, all what can contribute to optimisation of the gas transportation system. Determination of the characteristics is based on the laws of thermodynamics.

The gas on gas pipelines is compressed by gas pumping units (GPU) at the compressor stations (CS) mounted every ten kilometres of the pipeline depending on the performance of the gas pipeline [3] [4]. Gas mains usually have the diameters of pipes from 700 to $1400 \mathrm{~mm}$, a capacity of 50 to 90 million $\mathrm{m}^{3}$ of gas per day. GPU consist primarily of a gas turbine drive to drive the gas centrifugal compressor, which compresses gas.

\section{The Calculated Ratios of Thermodynamics for Gas Compression}

Based on the thermodynamics of the gas flow, the potential work of the gas is equal to the work in the gas transfer from the area of one pressure $\left(P_{1}\right)$ to the other $\left(P_{2}\right)$. This work is influenced by the potential difference [5].

$$
W_{1,2}=-\int_{1}^{2}(v \mathrm{~d} p)=-\int_{1}^{2}(\mathrm{~d} p / \rho)=(p / \rho)_{m} \ln \varepsilon,
$$

where $\varepsilon$ is the ratio of pressures in the compression or expansion, $\mathrm{m}$-hereinafter the index of average values; $(p / \rho)_{m}=\left[(p / \rho)_{1}+(p / \rho)_{2}\right] / 2$, where $\rho$ is gas density, a value reciprocal of the specific gas volume $(v)$ (hereinafter, the dimensions of all values in formula are given in SI units). Expression of the potential work applies to all types of flow machines and is the most important energy characteristic.

The product of two functions of the states, the absolute pressure and specific volume form a new feature of the state of gas $p v=p / \rho$ called the potential function successfully used in calculations when calculating the work.

The reversible potential work in a given pressure range is independent of the type of machine (sequencedflow machines, impeller machines, blade machines or high-volume machines, piston machines); in particular in nozzles and blades of the respective machines the potential work is related to the conversion of kinetic energy into potential in the compression machines: pumps, compressors, blowers etc.; and in expansion machines like turbines, on the contrary, the potential energy of the working medium with a high temperature and high pressure in the flow part of the machines transforms into the kinetic energy. The potential work is graphically and numerically determined by the area under the curve of the process 1 - 2 in the coordinates $P$ - $v$ (specific volume of gas), and the area facing the $y$-axis. The potential work is a reversible work of flow, positive in the expansion process $(\Delta P>0)$ or negative in the compression process $(\Delta P<0)$.

The index of any thermodynamic process ( $n$-the first average polytropic index) is determined as the ratio of the potential and thermodynamic work:

$$
n=w_{1,2} / l_{1,2},
$$

where $l_{1,2}$-the thermodynamic work of change in the volume of closed systems. The estimated expression of the work of any thermodynamic systems:

$$
w_{1,2}=n l_{1,2}=n p_{1}\left(1-\tau_{1,2}\right) /(n-1) \rho_{1},
$$

where $\tau_{1,2}$ is a characteristic of expansion (compression), as the ratio of the potential functions of the boundary points of the process, is the transformed equation of any thermodynamic process:

$$
\tau_{1,2}=p_{2} \rho_{1} / p_{1} \rho_{2}=\left(p_{2} / p_{1}\right)^{(n-1) / n}=\left(\rho_{2} / \rho_{1}\right)^{n-1}=z_{2} T_{2} / z_{1} T_{1},
$$

where $z, T$ - compressibility factor and gas temperature.

The nature of the distribution and emergence of the potential work as well as the work of any thermodynamic system is based on the law of conservation of mechanical energy of the reversible and real systems.

The potential work in the range of change of pressure, for example, an infinitesimal value $d p$ is applied to the performance of the external and effective work $\delta w^{*}$ and for the work of irreversible energy losses $\delta w^{* *}$ (hereinafter the sign $\delta$ denotes the infinitesimal quantities which are functions of the process, for example, the work $w$, 
heat exchange $q$ )

$$
\delta w=-v d p=\delta w^{*}+\delta w^{* *} .
$$

This ratio characterises the nature of distribution of the potential work in its first stage. Then, at the second stage, the external and effective work is allocated to the work directly transferred the bodies of the external system $\left(\delta w_{c z}^{*}\right.$ ) (for example, on the shaft of the gas turbine, the positive work by size, or negative the work taken from the outside to the centrifugal gas compressor, the work on the coupling), and the change in the energy of the external position of the working medium $\left(d e_{c z}\right)$. The last term of sum in turn, consists of the kinetic energy $d$ $\left(c^{2} / 2\right)$ and potential energy $(g d z)$.

Finally, in a general form the equation of occurrence and distribution of the flow work can be represented by the following expression:

$$
\delta w=-v d p=\delta w_{c z}^{*}+d\left(c^{2} / 2\right)+g d z+\delta w^{* * *} .
$$

A sub-expression of the presented principle of the occurrence and distribution of the potential flow work is the well-known Bernoulli equation, which can be obtained from (6). One of the common expressions of Bernoulli's equation in the form of the so-called "sum of three heights" (where head is in metres) conceals the true physical meaning of the equation emergence and spread of the potential flow work (5), (6); In addition, this equation includes the non-systemic and not thermodynamic value, i.e. the specific weight depending on the acceleration of gravity at the point of weighing.

\section{Determination of the Potential Work and Power Used to Compress Gas in the Gas Transport through Pipelines}

We present the calculated expression for the potential work of gas compression in the gas centrifugal compressors GPU of the gas compressor units of gas pipelines as the equation of occurrence of gas flow work from the formula (6) in an integral form:

$$
w_{1,2}=-\sum_{1}^{2} v d p=R T_{m} \sum_{1}^{2} z_{m} d p / p=R T_{m} \sum_{p_{p r 1}}^{p_{p r 2}}\left(z d p_{p r} / p_{p r}\right) .
$$

An analysis of the main gas state parameters during its transportation shows that its volume and pressure significantly change, and temperature changes to a lesser extent. This means that averaging the compressibility factor may lead to certain errors. Considering the above, the initial formula (7) is integrated taking into account only averaging the temperature $T_{m}$.

When studying the compressibility of natural gas as a mixture of the various components, the experimental results are processed the same way as in the studies of individual single-component gases, but $z$ is not determined by the reduced parameters but the pseudo-reduced gas mixture parameters:

$$
\begin{aligned}
& z=f\left(p_{p r}, T_{p r}\right) ; \\
& p_{p r}=p / p_{p c r} ; T_{p r}=T / T_{p c r} ; \\
& p_{p c r}=\sum_{i=1}^{n}\left(r_{i} p_{c r i}\right) ; T_{p c r}=\sum_{i=1}^{n}\left(r_{i} T_{c r i}\right),
\end{aligned}
$$

where $p_{p r}$ is the pseudo-reduced pressure, and $T_{p r}$ is the pseudo-reduced temperature of the mixture of gases; $p_{p c r}$ is the pseudo-critical pressure of the gas mixture; $T_{p c r}$ is the pseudo-critical temperature of the gas mixture; $r$ is the molar (voluminous) weight-part concentration of various components of the mixture; $p_{c r i}, T_{c r i}$ are the critical parameters of various components of natural gas, the number of which varies from 1 to $n(i=1,2, \cdots, n)[6]$.

In [7] provided is a number of design formulas to determine the compressibility factor depending on the pseudo-reduced parameters from the known literature [7] [8].

The results of calculations of the pseudo-reduced parameters by correlation equations obtained by VNIIGAZ (Russian Research Institute for Natural Gases and Gas Technologies) within the accuracy of up to (0.3 - 0.5)\%:

$$
p_{p r}=47.9-\Delta b a r ; T_{p r}=162.8(0.613+\Delta)^{\circ} \mathrm{K},
$$


where $\Delta$ is the specific gravity of gas.

In the practice of thermodynamic calculations the natural gas compressibility factor is determined from dependence tables or diagrams $z=f\left(p_{p r}, T_{p r}\right)$ or $z=f(p, t, \Delta)$ as well as by various empirical formulas valid for specific conditions set forth in Table 2 [7] [8].

\section{Initial Data}

To determine the potential operation of gas compression natural gas by its composition similar to the natural gas of gas fields was adopted (see Table 1 )

According to the composition of the natural (see Table 1) the parameters of the critical state of gas are calculated. The calculation results are presented in Table 2.

The calculated results by determining the potential work of gas compression in the gas centrifugal compressor GPU of the gas compressor units of gas pipelines following the above formulas (7) - (11) are shown in Table 3.

According to the data of Table 3, in the case of non-total-head gas transport, the centrifugal gas compressor GPU of the compressor units of gas pipelines in the transport of gas of 50 million $\mathrm{m}^{3} /$ day, the capacity of 17,880 $\mathrm{kW}$ is used while in the case of 90 million $\mathrm{m}^{3} /$ day significantly more, $32,180 \mathrm{~kW}$.

In the context of the declining productivity of gas from the fields, the power spent on gas transportation increases significantly. Thus, in the case of full-head gas centrifugal compressors GPU of the compressor units of gas pipelines with a degree of compression of 2 with the productivity of 50 million $\mathrm{m}^{3} /$ day the capacity is up to $63,020 \mathrm{~kW}$, and at 90 million $\mathrm{m}^{3} /$ day it increases to $113,440 \mathrm{~kW}$.

Table 1. Composition of natural gas.

\begin{tabular}{ccccccc}
\hline & & \multicolumn{3}{c}{ Gas composition } \\
\cline { 2 - 6 } Parameter & Methane & Ethane & Propane & Isobutane & Normal butane & CO \\
Molar share concentration $r_{i}$ & 0.9432 & 0.0390 & 0.0117 & 0.0008 & 0.0013 \\
Molar weight $\mu_{i}(\mathrm{~kg} / \mathrm{mol})$ & 16 & 30 & 44 & 58 & 58 \\
\hline
\end{tabular}

Table 2. The value of parameters of the critical state of gas.

\begin{tabular}{|c|c|c|c|c|c|}
\hline Component & $r_{i} \mu_{i}(\mathrm{~kg} / \mathrm{kmol})$ & $T_{c r}(\mathrm{k})$ & $r_{i} T_{c r i}$ & $p_{c r}(\mathrm{bar})$ & $r_{i} p_{c r i}$ \\
\hline Methane & 15.09 & 191 & 179.2 & 47.1 & 44.4 \\
\hline Ethane & 1.17 & 305 & 11.9 & 49.5 & 1.9 \\
\hline Propane & 0.51 & 370 & 4.3 & 43.2 & 0.5 \\
\hline Isobutane & 0.04 & 408 & 0.4 & 36.9 & 0.03 \\
\hline Normal butane & 0.07 & 425 & 0.6 & 38.6 & 0.05 \\
\hline $\mathrm{CO}_{2}$ & 0.17 & 305 & 1.2 & 75.2 & 0.3 \\
\hline Mixture parameters, total & 17.05 & --- & 197.6 & --- & 47.2 \\
\hline
\end{tabular}

Table 3. The value of the potential work depending on the ratio of pressure (degree of compression) during gas compression.

\begin{tabular}{|c|c|c|c|c|c|c|c|c|c|c|}
\hline \multirow{2}{*}{$\begin{array}{l}\text { Number of the } \\
\text { calculation formula }\end{array}$} & \multicolumn{10}{|c|}{ Degree of compression } \\
\hline & 1.1 & 1.2 & 1.3 & 1.4 & 1.5 & 1.6 & 1.7 & 1.8 & 1.9 & 2.0 \\
\hline (7) & 14.059 & 26.215 & 36.613 & 46.864 & 55.797 & 64.731 & 72.346 & 79.522 & 86.259 & 92.41 \\
\hline (7) & 191.76 & 357.6 & 499.40 & 639.22 & 761.07 & 882.93 & 986.81 & 1084.68 & 1176.57 & 1260.47 \\
\hline
\end{tabular}

Note: The numerator gives the value of the potential work in $\mathrm{kJ} / \mathrm{kg}$, in the denominator $\mathrm{kW} /\left(\right.$ million $\left.\mathrm{m}^{3} / \mathrm{day}\right)$. 
According to the above data we can calculate the power consumed along the whole gas main, for example, with 20 compressor plants with centrifugal compressors. With the pipeline productivity of 50 million $\mathrm{m}^{3} /$ day in the full-head mode power, the capacity costs amount to 1,260,400 kW, and they reach 2,268,800 kW at 90 million $\mathrm{m}^{3} /$ day.

\section{Conclusion}

The article presents the basic calculation ratios for the compression process GPU of compressor plants of gas pipelines to determine the potential performance and power consumed to compress the gas at compressor plants in the transport of real gases at pseudo-critical and pseudo-reduced parameters for natural gas. The characteristics of natural gas in its composition similar to the natural gas of gas fields are taken as the initial data. According to the above data, we can calculate the power consumed along the whole gas main.

\section{References}

[1] Belokon, N.I. (1968) Osnovnye printsipy termodinamiki. Nedra Publ., Moscow, 110.

[2] Mikaelian, E.A. and Mouhammad, S.A. (2015) Survey of the Gas-Turbine Gas-Compressor Units of Compressor Plants Based on the Modified Thermodynamics Model. International Journal of Alternative Fuels, 17, 1125-1131. http://recentscience.org/ijaf-international-journal-of-alternative-fuels/

[3] Vladimirov, A.I. and Kershenbaum, Y.V. (2008) Industrial Safety Compressor Stations. Management of Safety and Reliability. Inter-Sector Foundation "National Institute of Oil and Gas”, Moscow, 640. http://www.mdk-arbat.ru/bookcard?book_id=3304125

[4] Mikaelian, E.A. and Mouhammad, S.A. (2015) Thermodynamics of Efflux Process of Liquids and Gases. Journal of Power and Energy Engineering, 3, 71-75. http://dx.doi.org/10.4236/jpee.2015.35006

[5] Heppenstall, T. (1998) Advanced Gas Turbine Cycles for Power Generation. Applied Thermal Engineering. 18, 837846. http://dx.doi.org/10.1016/S1359-4311(97)00116-6

[6] Kling, R. (1980) Thermodynamique générale et Applications. Editions Technip, Paris. http://www.worldcat.org/title/thermodynamique-general-et-applications/oclc/301431244/editions?referer=di\&editions View=true

[7] Mikaelian, E.A. (2000) Tekhnecheskoe obuslogivani energotekhnologicheskovo oborudivani, gazoturbinnykh perekachivayushchikh agregatov sestemi cobra e transporta gasa (Maintenance of the Power Engineering Equipment, Gas-Turbine and Gas-Compressor Units of the Gas Collection and Transfer Systems). Methodology, Research, Analysis and Practice, Fuel and Energy, Moscow, 314. http://www.dobi.oglib.ru/bgl/5076.html

[8] Katz, D.L., Cornell, D., et al. (1965) Rokovodstvo po dobeche, transporto I pererebotke prirodnovo gaza. Translate from English, Nedra Publ., Moscow, 676. 